\title{
Fuzzy Logic Decision based Adaptive Directional Weighted Median Filter for Restoring Impulse Corrupted Images
}

\author{
S. Abdul Saleem \\ Research Scholar \\ Jamal Mohamed College, Tiruchirappalli \\ Tamil Nadu, India
}

\author{
T. Abdul Razak, PhD \\ Associate Professor in Computer Science \\ Jamal Mohamed College, Tiruchirappalli \\ Tamil Nadu, India
}

\begin{abstract}
Image restoration and enhancement are the major research areas in digital image processing. The main objective of image restoration is to reduce noise and improve resolution loss on digital images in any real-time domains. Many images like photographs, medical images, satellite images and aerial images suffer from poor contrast and noises due to various reasons such as lightening, bad weather or flaw in the equipment. It is necessary to restore the image by removing impulse noises and to increase the image quality by using image parameters. Number of image restoration filters have been introduced in the past decades and tested on standard images to prove their efficiency. This study proposes a new fuzzy logic decision based adoptive directional weighted median filter for the restoration of impulse corrupted digital images. The proposed filter includes fuzzy logic based decision to model the uncertainties, while detecting and correcting impulses. The proposed correction scheme provides weight to the uncorrupted pixels that show much similarity with other uncorrupted pixels in the $3 \times 3$ kernel window while replacing impulses. The proposed fuzzy filter adapts to various noises level and image conditions and is capable of suppressing noise while preserving image details. The experimental outcome in terms of subjective and objective metrics favours the proposed algorithm than many other major filters in the literature.
\end{abstract}

\section{General Terms}

Image restoration, impulse noise, spatial domain, directional weighted median, quantitative metrics

\section{Keywords}

Gaussian noise, salt \& pepper noise, image parameters, edge preservation, fuzzy logic

\section{INTRODUCTION}

Impulse noises affect images when they are captured by faulty sensors or when they are transmitted through unsecured communication channels. Salt and pepper is a type of impulse noise which corrupts randomly selected pixels of digital image into a very large value as positive impulse or a very small value as negative impulse [1]. Since this noise affects the true content of the digital image, the challenge in the image preprocessing domain is to efficiently reduce the effect of impulse noise while preserving the image details. Median filters are the popular non-linear filters widely applied to remove salt and pepper noises because of its computational efficiency and simplicity. The standard median filter (SMF) uniformly replaces the gray values of all pixels in the digital image irrespective of whether they are corrupted or not by the median of respective pixels in the static neighborhood and provides good noise suppression since the noise pixels fall in extreme ranges of a sorted array while determining median. The position-invariant median filter and its variants during restoration consume image details while removing impulses [2]. Rank ordered adaptive median filter (ROAMF) is proposed by Hwang, H. et al., which keeps the image details of highly corrupted digital images by switching the filtering of only the corrupted signals with a median value chosen from a neighborhood that varies adaptively with the quantum of impulse noise. Adaptive median filter (AMF) detects corrupted signals by checking them to be between minimum and maximum of the median detected neighborhood, it fetches a dependable median from an adaptively varying neighborhood for only the corrupted signals and works very well for all types of digital images up to $60 \%$ noise levels[1][3]. The main limitation of this filter is that, the impulse replacing median is not determined from uncorrupted pixels, impulse replacing median from a bigger window affects the image fidelity, unnecessary increase of windowsize though uncorrupted pixels are in a smaller window and computationally this filter is costly. Moreover, it does not attend to any corruption check for the determined median before replacing the pixel values. These drawbacks met by median filter are addressed by many next generation filters like adaptive switching median filter (ASMF) by Nallaperumal, K. et al. [8] , noise adaptive switching median filter (NASMF) by Kenny Kal Vin Toh et al. [9], Decision Based Algorithms by Srinivasan, K.S. et al., Nair Madhu, S, et al. [11][12] and fuzzy decision based switching median filter (FDSMF) by Saleem, S. et al. [14] introducing decision or switching schemes during the filtering phase. As an extension to NASMF, NAFSMF, the noise adaptive fuzzy switching median filter (NAFSMF) utilises the histogram of the corrupted image to identify noisy pixels and later uses fuzzy reasoning to restore the correct pixel on the impulses that are detected. However, the noise detection stage is very simple and the possibility of misclassification of uncorrupted pixels as impulses is more, the filter proposed by Xu et al.[15] use an $\mathrm{S}$ function to fuzzify the amount of corruption of individual pixels. Since, the filter could not provide sufficient restoration since there is no adaptation in the window size, Numerous other filters [3-7] also introduced with different switching and optimization schemes by meeting some requirements of impulse noise filtering but failed to simultaneously meet on other vital requirements like the computational efficiency, misclassification of pixels, maintaining fidelity in restored outputs and so on.

This paper proposes a new Fuzzy logic decision-based adaptive directional weighted median (FDADWM) filter for the detection and correction of salt and pepper impulse noises. The proposed filter also concentrates to maintain fidelity in the restored images. This paper is organised in six sections. 
Section 2 introduces the new FDADWM filter; Section 3 provides the experimental methodology used for comparing the performance of the proposed filter. The comparative analysis on the subjective and objective metrics of the proposed filter with other important filters is presented in Section 4. Section 5 gives the conclusion and future direction of the work.

\section{FDADWM FILTER}

The proposed approach works in two distinct stages of impulse detection and impulse correction as shown in Fig. 1.

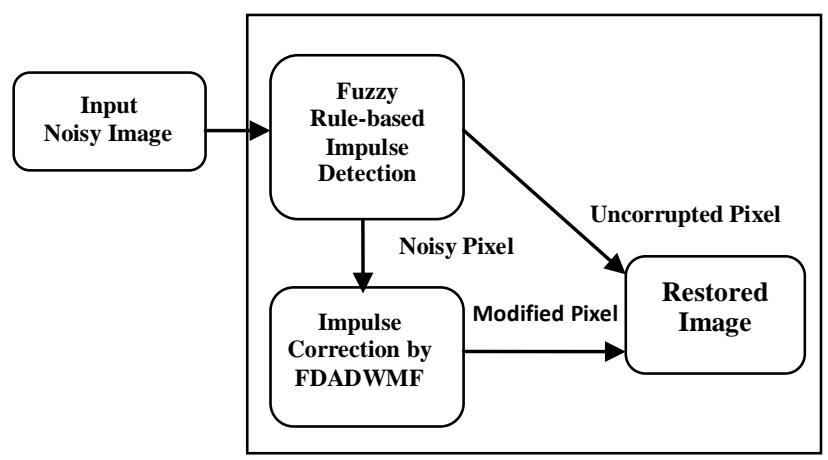

Fig. 1: Block Diagram of Proposed Approach

\subsection{Impulse Detection Stage}

In the first stage, a fuzzy flag image $\mathrm{f}$ is created to indicate the amount of corruption of each pixel in the given corrupted image of same size as the input image $X$ of size $m \times n$ to be filtered where $f_{i}$ and $x_{i}$, respectively, denote the flag value and pixel value at position $x=(i, j)$. Set $f_{i}=0$ to indicate the pixel value at spatial position $x$ to be an impulse and $f_{i}=1$ when the pixel value at $x$ is not impulse. Initially this flag image, $\mathrm{f}$ is set to ' 0 ' at all its spatial locations assuming that all the pixels of the image, $X$ are fully corrupted. $f_{i}$ is the fuzzy membership value allotted to the pixel at position $\mathrm{x}$ to indicate the impulsive or uncorrupted nature of the pixel. The value of $f_{i}$ ranging from 0 to 1 is the degree of the purity of the pixel that varies from impulsive to uncorrupted. An impulse corrupted pixel has characteristics very similar to that of an edge pixel. So, $\mathrm{f}_{\mathrm{i}}$ is determined through the following steps by using the fuzzy rules defined on minimum directional differences. The impulse detection scheme can be tracked through the following steps:

Step 1: The set of noise free pixels, $S_{1}$ and the window size, $\mathrm{W}$ for analysis of pixels is identified. Set $\mathrm{W}=3$, since it reduces the computational complexity and there is additional refinement step to reduces the misclassifications of pixels.

Step 2: The set of spatial positions within a square window $3 \times 3$, centered at each pixel position, $x=(i, j)$, is defined by the set, Here, $\mathrm{W}$ indicates the size of the local neighbourhood window under consideration. The phase begins by analysing the pixel-wise characteristics of the corrupted image, $\mathrm{X}$ in the local neighbourhood, $3 \times 3$.

Step 3: Check the purity status of $\mathrm{X}$ by comparing it with the minimum, M1 and maximum M2 as

$$
f_{x=(i, j)}= \begin{cases}1 & M 1<x_{i}<M 2 \\ 0 & \text { otherwise }\end{cases}
$$

where

$$
M_{1}=\min \left\{x_{i} \in \quad W_{K}\right\}
$$

$$
M_{2}=\max \left\{x_{i} \in W_{K}\right\}
$$

Step 4: Fuzzify the purity status of $X_{i}$ when $X_{i}$ is found not to lie strictly in between $M_{1}$ and $M_{2}$ since edge pixels are also having similar properties. To estimate the amount of corruption of detected corrupted pixels, the uncorrupted pixels in the neighbourhood around $\mathrm{Xi}$ are caught in the impulse-free pixel set, $\mathrm{S}_{1}$, that is,

$$
S_{1}=\left\{x_{j} \in W_{3} \text { and } M_{1}<x_{j}<M_{2}\right\}
$$

Step 5: If the cardinality of uncorrupted pixels, $S_{1}$ is greater than a predefined threshold, $T_{1}$, it is easy to find whether the current pixel under consideration is an edge pixel similar to the noise. Hence, find the directional distances, $d_{k}$ in each direction, $\mathrm{k}$ as defined in Fig. 2
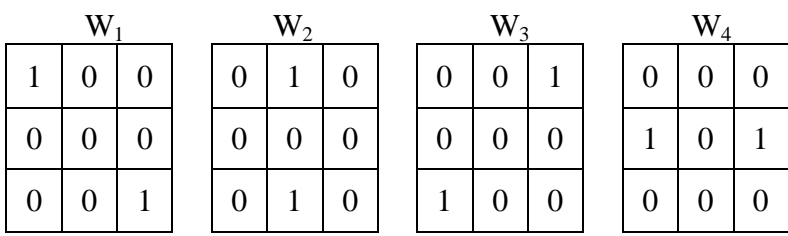

Fig. 2 : Directional Weights

$d_{k}=\sum_{k=1}^{k=4} \operatorname{Mask}\left(W_{k}\right) * \operatorname{abs}(\mathrm{a}(\mathrm{i}, \mathrm{j})-\mathrm{a}(\mathrm{i}+\mathrm{k}, \mathrm{j}+\mathrm{l}))$

Step 6: Lower values in any directional distances indicate the presence of an edge in the corresponding direction passing through the pixel under consideration. In order to model the uncertainty of the corruption status of these pixels, a fuzzy membership function SMALL is defined as in figure 3 . Accordingly, the membership of purity is formed as

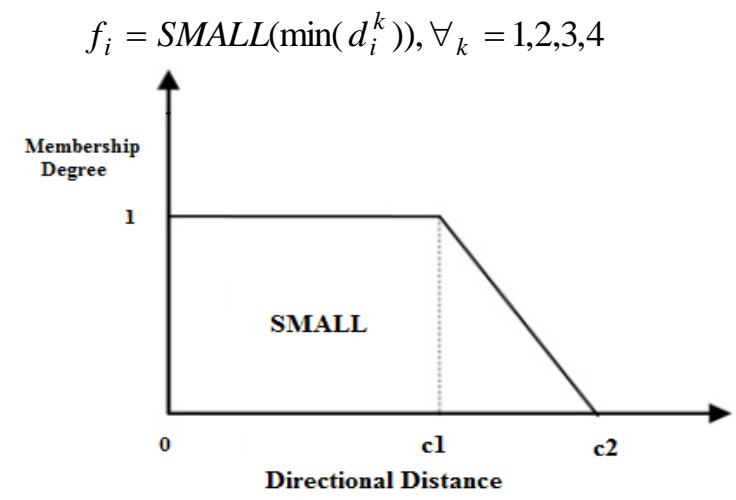

Fig. 3: Fuzzy Membership function SMALL

Step 7: Move to the next pixel for processing and repeat step 1 through step 7 until all pixels in the image are processed. Since the fuzziness incorporated by considering directional distances, the chances of escaping of edge pixels similar to that of impulse counterparts from wrongly detected as impulses are more.

As a part of the filtering mechanism in the proposed FDADWM filter, fuzzy reasoning is applied to the extracted local information

$D(i, j)$. The fuzzy set adopted is shown in figure 4 and defined by the membership function given in equation 7

$$
F(i, j)=\left\{\begin{array}{cc}
0 & D(i, j)<T_{1} \\
\frac{D(i, j)-T_{1}}{T_{2}-T_{1}} & T_{1} \leq D(i, j)<T_{2} \\
1 & D(i, j) \geq T_{2}
\end{array}\right.
$$




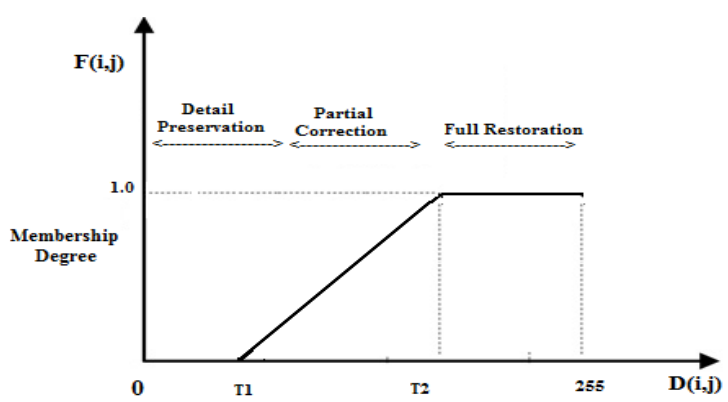

Fig. 4: Fuzzy Set adopted by FDADWMF

\subsection{Impulse Correction Stage}

The correction algorithm starts by taking the inputs of the flag image, $f$ and the input image, a. The algorithm process each pixel in the image at position $i=(i, j)$ such that $(i, j) \in$ Domain $(\mathrm{X})$ and can be tracked through the following steps.

Step 1: If $\mathrm{c}>0$ compute the median of non-impulsive pixels in the array $S_{1}$

Step 2: Obtain the maximum value in $\mathrm{S}_{2}$ (Distance between noise free neighboring pixel and central pixel ). If $\operatorname{Max}\left(S_{2}\right)$ $<=20$ set flag1 to 0. Suppose $\operatorname{Max}\left(\mathrm{S}_{2}\right)<=50$, compute flag1 as $\left(\operatorname{Max}\left(\mathrm{S}_{2}\right)-20\right) / 30$ otherwise flag1 is set to 1

Step 3: If $\mathrm{c}=0$ Compute the average of previously processed pixels $b(i-1, j)$ and $b(i, j-1)$ and taken as median value.

Step 4: Compute $\mathrm{b}=(1-\mathrm{flag} 1)^{*} \mathrm{a}(\mathrm{i}, \mathrm{j})+$ flag $1 *$ median of $\mathrm{S}_{1}$ or average of previously processed pixels depending upon the value of $\mathrm{c}$.

Step 5: Repeat the above steps for all the pixels in the image

\section{EXPERIMENTAL METHODOLOGY}

The proposed FDADWM filter implemented and tested by using sample version of MATLAB image processing tool on a variety of digital images of which the Lena, Cameraman, Peppers and Car images of size $256 \times 256$ are used in this paper for objective and subjective comparisons with the results of top-ranking other nonlinear non-fuzzy and fuzzy filters: the standard median filter (SMF), rank ordered adaptive median filter (ROAMF) [1][5], the decision based algorithms (DBA) [11][12], Noise Adaptive Fuzzy Switching Median Filter (NAFSMF) [9] and Fuzzy Logic Decision Based Adoptive Switching Median Filter (FDASMF) [14]. The quantitative metrics used for analysing the improved performance of the proposed impulse detection and correction stages of FDADWMF are the Peak Signal-to-Noise Ratio (PSNR), the Mean Square Error (MSE), the Root Mean Square Error (RMSE), Image Enhancement Factor (IEF), Time Factor and Structural Similarity Index Metrics (SSIM) of the restored image are given in equation 8-12.

$$
\begin{gathered}
P S N R=10 \log _{10}\left(255^{2} / M S E\right) \\
M S E=(1 / M N) \sum_{i=1}^{M} \sum_{j=1}^{N}[O(m, n]-R(m, n)]^{2} \\
R M S E=\left[(1 / M N) \sum_{i=1}^{M} \sum_{j=1}^{N}[O(m, n]-R(m, n)]^{2}\right]^{1 / 2} \\
I E F=\frac{\sum_{i=1}^{M} \sum_{j=1}^{N}(N(m, n)-O(m, n))^{2}}{\sum_{i=1}^{M} \sum_{j=1}^{N}(D(m, n)-O(m, n))^{2}} \\
S S I M=\frac{\left(2 \mu_{O} \mu_{R}+C_{1}\right)\left(2 \sigma_{O R}+C_{2}\right)}{\left(\mu_{O}{ }^{2}+\mu_{R}{ }^{2}+C_{1}\right)\left(\sigma_{O}{ }^{2}+\sigma_{R}{ }^{2}+C_{2}\right)}
\end{gathered}
$$

Where, $\mathrm{O}$ is the original image; $\mathrm{R}$, the restored image; $\mathrm{D}$, denoised image; $\mu_{O}$ and $\mu R$ are the averages of $O$ and $R$ respectively; $\sigma_{O}{ }^{2}$ and $\sigma_{R}{ }^{2}$ are variances of $O$ and $R$ respectively; $\sigma_{\mathrm{OR}}$ is the correlation coefficient between $\mathrm{O}$ and $\mathrm{R} ; \mathrm{C}_{1}$ and $\mathrm{C}_{2}$ are small constants for stabilize the computation; $\mathrm{C} 1=\left(\mathrm{k}_{1}+\mathrm{L}\right)^{2} \quad \mathrm{C} 2=\left(\mathrm{k}_{2}+\mathrm{L}\right)^{2} \quad \mathrm{k}_{1}=0.01$ and $\mathrm{k}_{2}=0.03$ by default; $\mathrm{L}=255$.

\section{EXPERIMENTAL RESULTS AND ANALYSIS}

The image details-preserving aspect of the proposed filter together with other top-ranking filters is analysed through the visual comparison of the restored outputs in Figures 5 and 6 and also emphasised the edge preservation of the restored outputs from Car image corrupted with $65 \%$ noise level for NAFSMF, FDASMF and the proposed ADADWMF filters are shown in Figure 7(b), 7(c) and 7(d) respectively, but 7(a) represents the edges produced from noise-free Car image. The subjective comparison made in Figures 5 and 6, respectively, on images Car and Peppers for impulse noise densities $15 \%$, $55 \%$ and $95 \%$ favour the proposed ADADWMF over the other top-ranking filters. From Figure 7, it is very clear that proposed ADADWMF provides better edge preservation than other filters. More edge details are retained in the restored outputs of the proposed filter. The objective comparison made in Tables 1 and Table 2 for Car and Peppers images record the metrics, PSNR, MSE, MAE, RMSE, IEF, Time and SSIM for the proposed $\mathrm{ADADWM}$ and other top-ranking filters for impulse noise densities $15 \%, 55 \%$ and $95 \%$. It is noted that at all noise densities, the objective and subjective metric values produced by the proposed filter outperform the comparative filters in terms of impulse detection, noise removal and details-preservation.

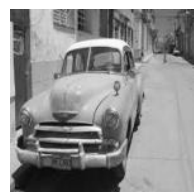

(a)

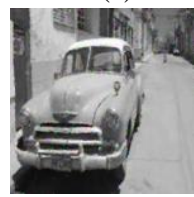

(e)

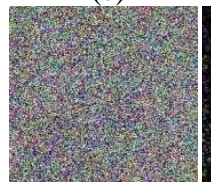

(i)

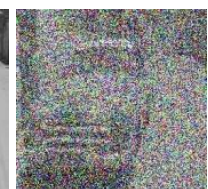

(b)

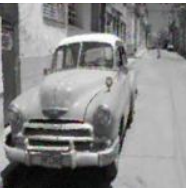

(f)

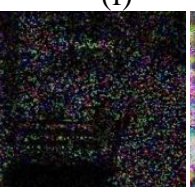

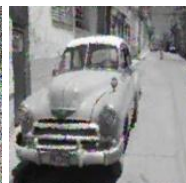

(c)

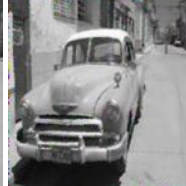

(g)

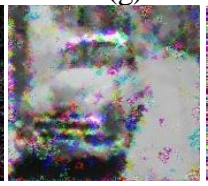

(k)

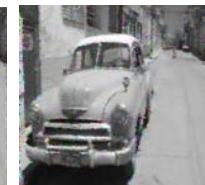

(d)

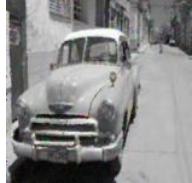

(h)

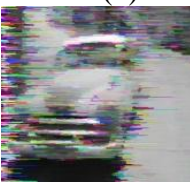

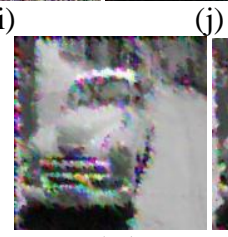

(m)

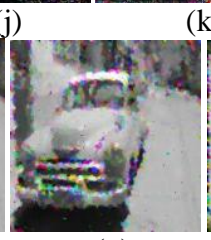

(n)

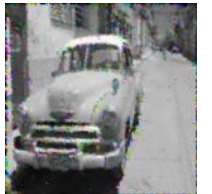

(o)
Fig. 5 : (a) Gray-Scale Car Image (b) Car image with $15 \%$ Noise Density and same image restored with (c) SMF, (d) ROAMF, (e) DBA, (f) NASMF, (g) FDASMF and (h)Proposed Algorithm (i) Gray-Scale Lena Image with $95 \%$ Noise Density and same image restored with $(\mathrm{j})$ SMF, (j) ROAMF, (k)DBA, (l) EASMF, (m)NAFSMF and (o)Proposed Algorithm 


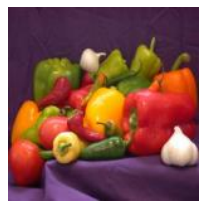

(a)

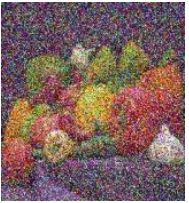

(b)

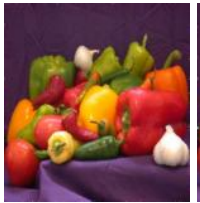

(c)

(d)

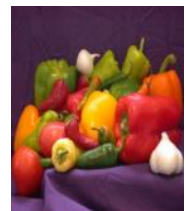

(e)

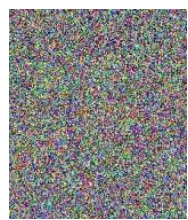

(i)

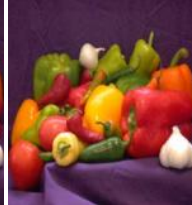

(f)

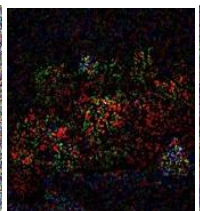

(j)

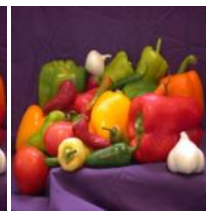

(g)

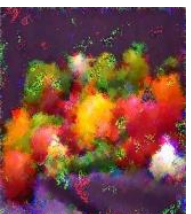

(k)

(h)

(1)
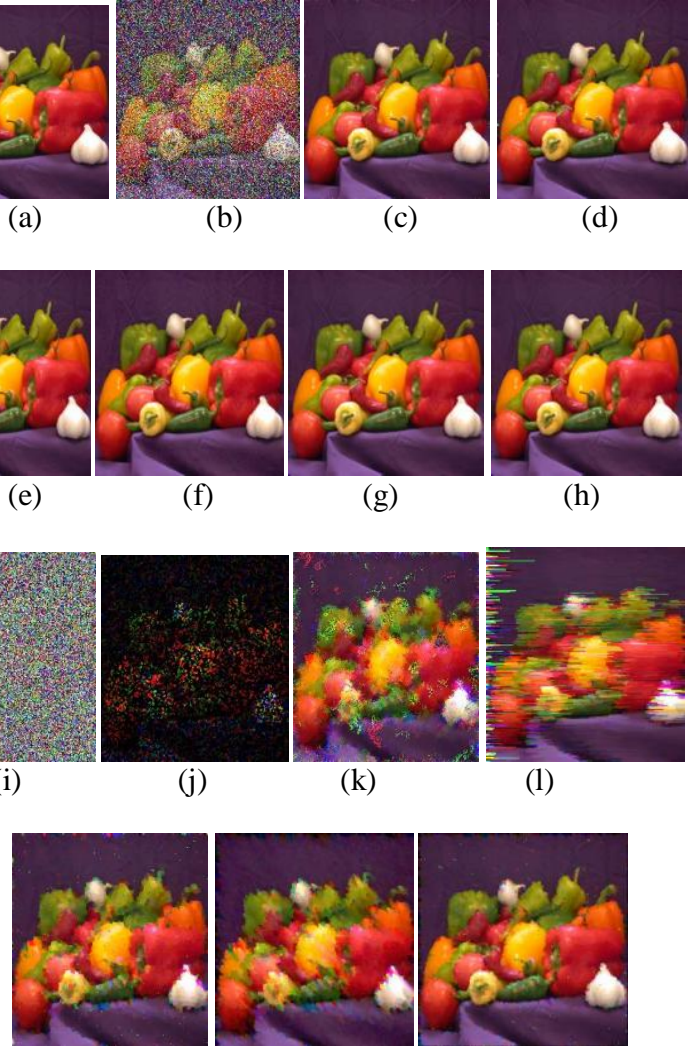

(m)

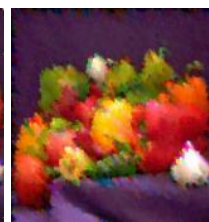

(n)

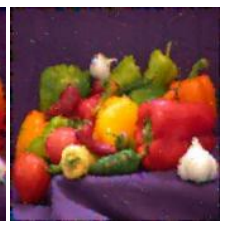

(o)
Fig. 6: (a) Color Peppers Image (b) Peppers image with $15 \%$ Noise Density and same image restored with (c) SMF, (d) ROAMF, (e) DBA, (f) NASMF, (g) FDASMF and (h)Proposed Algorithm (i) Color Peppers Image with 95\% Noise Density and same image restored with (j) SMF, (j) ROAMF, (k)DBA, (l) EASMF, (m) NAFSMF and (o)Proposed Algorithm

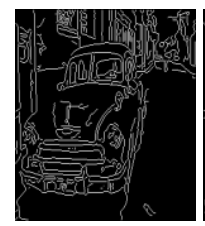

(a)

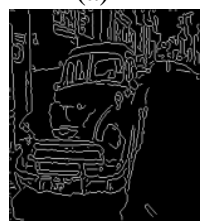

(e)

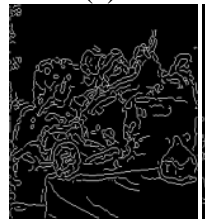

(i)

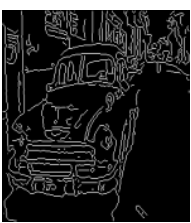

(b)

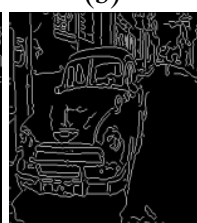

(f)

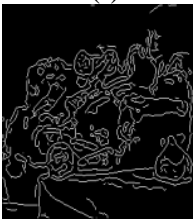

(j)

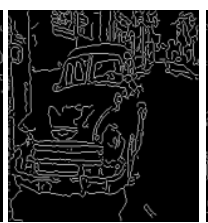

(c)

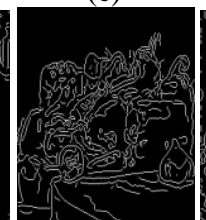

(g)

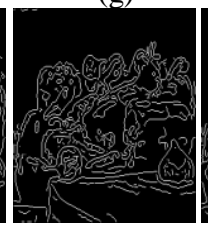

(k)

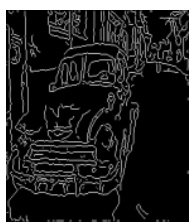

(d)

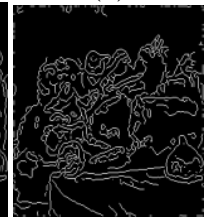

(h)

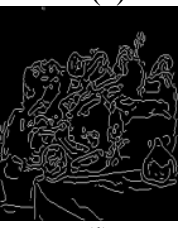

(1)
Fig. 7 : The edge detected image of the restored outputs of Car and Peppers images corrupted with $65 \%$ noise level for ROAMF, DBA, NAFSMF, FDASMF and proposed FDADWMF filters respectively and (a) \& (g) represent the edges produced from noise-free Car and Peppers images

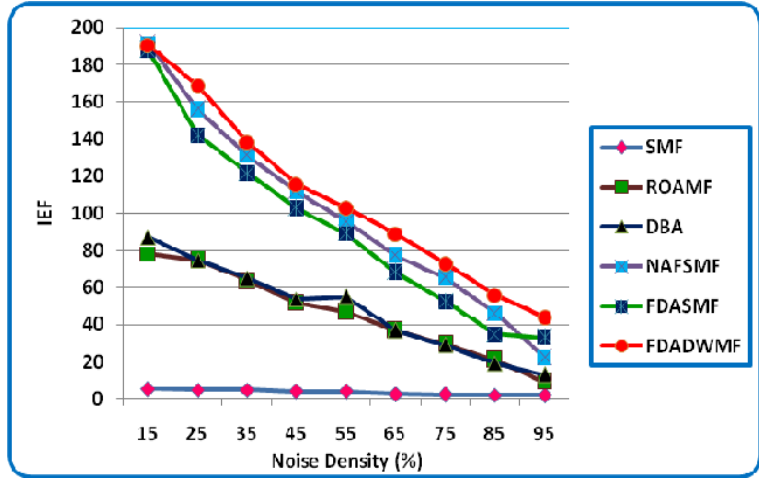

Fig. 8: Noise Density versus IEF of Car Image

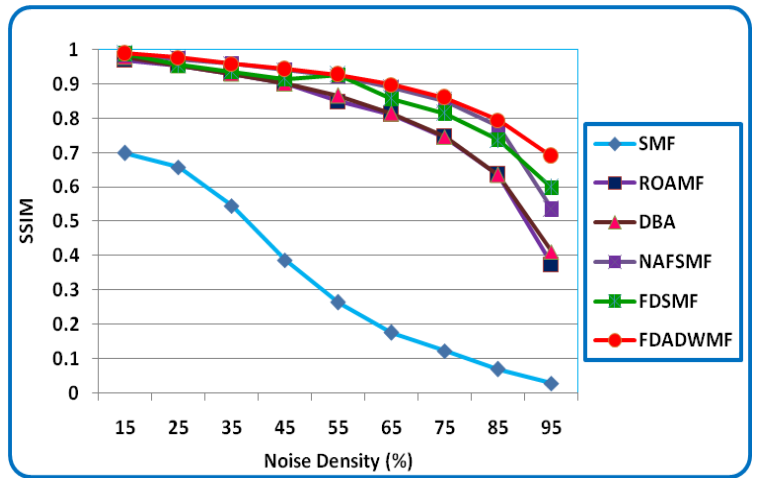

Fig. 9: SSIM versus Noise Density of Car Image

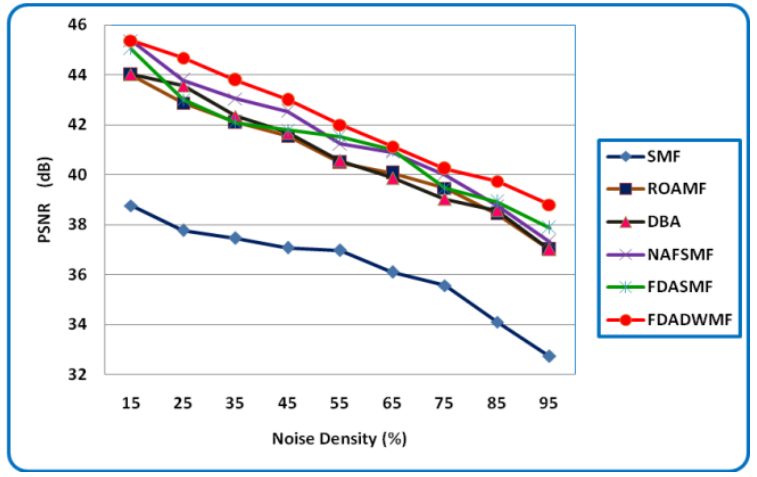

Fig. 10: PSNR versus Noise Density of Peppers Image

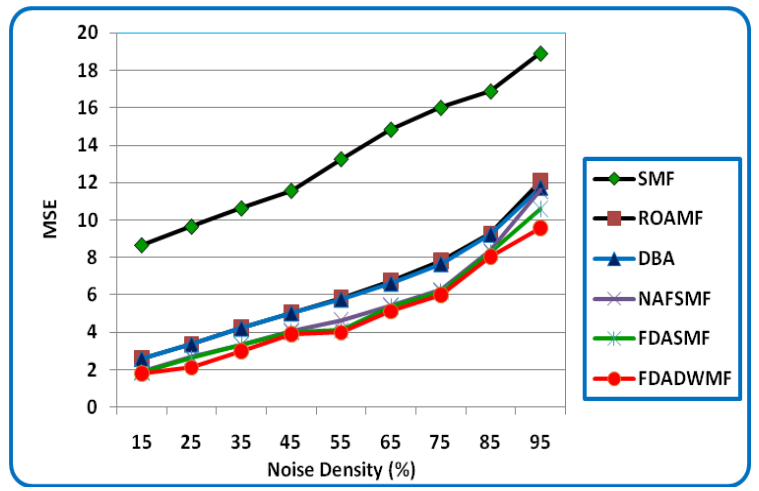

Fig. 11: MSE versus Noise Density of Peppers Image 
Table 1. Comparison of PSNR, MSE, MAE, IEF, Time and SSIM values of different algorithms for gray-scale Car.jpg image at different noise densities

\begin{tabular}{|c|c|c|c|c|c|c|c|c|c|c|c|c|c|c|c|c|c|c|}
\hline \multirow{2}{*}{ Filters } & \multicolumn{6}{|c|}{ Noise Density : $15 \%$} & \multicolumn{6}{|c|}{ Noise Density : $55 \%$} & \multicolumn{6}{|c|}{ Noise Density : $95 \%$} \\
\hline & PSNR & MSE & MAE & IEF & Time & SSIM & PSNR & MSE & MAE & IEF & Time & SSIM & PSNR & MSE & MAE & IEF & Time & SSIM \\
\hline SMF & 37.687 & 11.076 & 4.201 & 5.226 & 4.305 & 0.6987 & 32.992 & 12.56 & 5.975 & 3.856 & 4.678 & 0.2632 & 31.811 & 14.286 & 7.286 & 1.891 & 4.85 & 0.0274 \\
\hline ROAMF & 41.905 & 7.123 & 1.471 & 77.99 & 2.079 & 0.9704 & 39.267 & 7.789 & 2.978 & 46.65 & 4.357 & 0.8497 & 36.98 & 13.056 & 6.469 & 8.986 & 25.78 & 0.375 \\
\hline DBA & 41.936 & 4.186 & 1.469 & 86.98 & 4.354 & 0.9789 & 39.287 & 7.666 & 2.901 & 54.89 & 4.356 & 0.8667 & 36.919 & 12.891 & 6.109 & 12.89 & 4.567 & 0.412 \\
\hline NAFSMF & 43.154 & 3.145 & 1.097 & 191.67 & 4.989 & 0.9867 & 39.583 & 6.866 & 2.456 & 95.56 & 5.648 & 0.9233 & 37.557 & 11.478 & 4.789 & 22.26 & 7.908 & 0.5355 \\
\hline FDADWMF & 43.897 & 1.983 & 1.083 & 190.33 & 5.234 & 0.9892 & 40.545 & 4.533 & 2.043 & 102.89 & 5.573 & 0.9281 & 39.434 & 8.878 & 3.678 & 43.89 & 5.902 & 0.6898 \\
\hline
\end{tabular}

Table 2. Comparison of PSNR, MSE, MAE, IEF, Time and SSIM values of different algorithms for Peppers.jpg color image at different noise densities

\begin{tabular}{|c|c|c|c|c|c|c|c|c|c|c|c|c|c|c|c|c|c|c|}
\hline \multirow{2}{*}{ Filters } & \multicolumn{6}{|c|}{ Noise Density : $15 \%$} & \multicolumn{6}{|c|}{ Noise Density : $55 \%$} & \multicolumn{6}{|c|}{ Noise Density : $95 \%$} \\
\hline & PSNR & MSE & MAE & IEF & Time & SSIM & PSNR & MSE & MAE & IEF & Time & SSIM & PSNR & MSE & MAE & IEF & Time & SSIM \\
\hline SMF & 38.7641 & 8.6432 & 3.1293 & 14.155 & 4.309 & 0.8166 & 36.967 & 13.25 & 5.874 & 12.24 & 4.430 & 0.355 & 32.7219 & 18.901 & 6.892 & 3.3981 & 4.654 & 0.0546 \\
\hline ROAMF & 43.9965 & 2.5908 & 1.0301 & 264.52 & 2.0599 & 0.9839 & 40.486 & 5.815 & 2.265 & 120.34 & 2.565 & 0.910 & 37.009 & 12.07 & 5.990 & 25.884 & 24.08 & 0.4448 \\
\hline DBA & 44.021 & 2.5768 & 1.0203 & 279.61 & 4.2627 & 0.9842 & 40.544 & 5.737 & 2.233 & 125.56 & 4.455 & 0.915 & 37.002 & 11.72 & 5.989 & 30.952 & 5.782 & 0.464 \\
\hline NAFSMF & 45.3727 & 1.8872 & 0.7625 & 651.25 & 4.1514 & 0.9923 & 41.225 & 4.629 & 2.199 & 305.87 & 12.507 & 0.926 & 37.312 & 11.58 & 3.995 & 42.523 & 17.419 & 0.6862 \\
\hline FDASMF & 45.0353 & 1.8396 & 0.7737 & 414.95 & 7.037 & 0.9976 & 41.525 & 4.098 & 2.005 & 319.98 & 6.679 & 0.936 & 37.875 & 10.62 & 3.924 & 44.022 & 11.345 & 0.5977 \\
\hline FDADWMF & 45.371 & 1.8013 & 0.7567 & 597.78 & 5.9282 & 0.9972 & 41.998 & 3.998 & 1.678 & 352.89 & 6.789 & 0.9495 & 38.801 & 9.569 & 3.215 & 52.515 & 9.655 & 0.7527 \\
\hline
\end{tabular}

\section{CONCLUSION AND FUTURE WORK}

The proposed FDADWMF filter is designed for the restoration of digital images that are corrupted with high density salt and pepper impulse noises. This filter can provide much acceptable and recognizable image restoration even with $5 \%$ of input signal and it can give better results at 35\%, $50 \%, 75 \%$ noise levels with an incomparable visual quality than other adaptive median filters. While the median filters such as SMF, ROAMF, DBA and improved DBA algorithms are give the better results in the quantitative metrics of up to $60 \%$ noise level, they produce impulse patches at the level greater than $60 \%$. Furthermore DBAs are produce horizontal and diagonal streaks at high noise density. But this filter eliminates the streaking effect by careful selection of neighboring pixels. The proposed filter is faster than RAMF and NAFSMF, since it uses a small and fixed window of size $3 \times 3$. In addition, it affects a smooth transition between the pixel values by utilizing the correlation between neighboring processed pixels while preserving edge details thus leading to better edge preservation. The proposed filter is tested from low to high noise densities up to $95 \%$ on different grayscale images and color images that yield recognizable and patches free restoration. The significant difference in quantitative metrics shown in graphs Figure 8 through 11 and visual perception with other competitive fuzzy and non fuzzy filters quantify a dominance of the proposed filter.

Even though, different kinds of noises normally anticipated in the communication images, the proposed filter is designed for the restoration of only salt and pepper impulse corrupted digital images. As a future work, the proposed filter can be extended to restore the digital images and digital videos corrupted by other impulse noise models that include the random valued impulse noise and the additive impulse noises. This can be tried by accommodating more intelligent hybrid soft computing techniques into the domain of impulse restoration. Furthermore, the optimisation of the matrices used in the proposed filter can be extended in the future.

\section{REFERENCES}

[1] Rafael C. Gonzalez, and Richard E.Wood. 2009. "Digital Image Processing”, 3rd Edition, Prentice-Hall.

[2] Wang, Z., Zhang, D.,1998, 'Restoration of Impulse Noise Corrupted Images using Long-Range Correlation', IEEE Signal Process. Lett., vol. 5, No. 1, pp. 4-7

[3] Wang, Z., Zhang, D.,1999, 'Progressive Switching Median Filter for the Removal of Impulse Noise from Highly Corrupted Images', IEEE Trans. Circuits Syst.-II, Analog Digit. Signal Process., 46, (1), pp. 78-80

[4] Ko, S.J. and Lee, Y.H. 1991, "Center weighted median filters and their applications to image enhancement", IEEE Trans. Circuits Syst., 38, pp. 984-993

[5] Hwang H. and Hadded R.A., 1995, "Adaptive Median Filter: New algorithms and results",IEEE Trans.Image Process, vol. 4, no. 4, 1995, pp. 499-502

[6] Kwame Osei Boating, Benjamin Weyori Asubam and David Sanka Laar, 2012 "Improving the Effectiveness of the Median Filter", International Journal of Electronic and Comunication Engineering, ISSN 0974-2166, vol. 5, pp. 85-97

[7] Zhang, X., Xiong, Y.,2009, 'Impulse noise removal using directional difference based noise detector and adaptive weighted mean filter',IEEE Signal Process. Lett., 16, (4), pp. 295-298

[8] Nallaperumal, K., Varghese, J., Saudia, S., 2006, 'Salt \& Pepper Impulse Noise Removal using Adaptive Switching Median Filter'. Proc. Conf. of Asia Pacific OCEANS'06, Singapore, pp. 1-8

[9] Kenny Kal Vin Toh, K., Mat Isa, N A., 2000, 'Noise Adaptive Fuzzy Switching Median Filter for Salt and Pepper Noise Reduction', IEEE Signal Process. Lett., 17, (3), pp. 281-284 
[10] Fitri, U., Uchimura, K., Koutaki, G.,2012, 'High Density Impulse Noise Removal by Fuzzy Mean Linear Aliasing Window Kernel', IEEE Int. Conf. Signal Process. Commun. Comput., pp. 711-716

[11] Srinivasan, K.S., and Ebenezer D., 2007, "A new fast and efficient decision based algorithm for removal of high density impulse noise", IEEE signal processing. vol. 14, no.3, pp. 189-192

[12] Nair Madhu S., Revathy K. and Tatavarti Rao, 2008, "Removal of Salt and Pepper Noise in Images: A new Decision-Based Algorithm "Proceeding of International Multi Conference of Engineers and Computer Scientists, vol. 1, pp. 19-21

[13] Soruba Marcell, J., Jayachandran, A., and Kharmega Sundararaj, G. 2012. "An Efficient Algorithm for Removal of Impulse Noise using Adaptive Fuzzy
Switching Weighted Median Filter", International Journal of Computer Technology and Electronics Engineering(IJCTEE), vol.2, issue 2, pp 1-8

[14] Abdul Saleem,S., Abdul Razak,T., 2015,"Fuzzy Logic Decision Based Effective Adaptive Median Filter for Removing High Density Impulse noises in Digital Images", International Journal of Applied Engineering Research (IJAER), vol. 10 No.82, pp. 84-90

[15] Xu, H., Zhu, G., Peng, H., Wang, D.,2004, 'Adaptive Fuzzy Switching Filter for Images Corrupted by Impulse Noise', Pattern Recognit. Lett., Vol. 25, (15), pp. $1657-$ 1661

[16] Fabijanska, A., Sankowski, D.,2011, 'Noise Adaptive Switching Median Based Filter for Impulse Noise Removal from Extremely Corrupted Images', IET Image Process., vol. 5, (5), pp. 472-480 